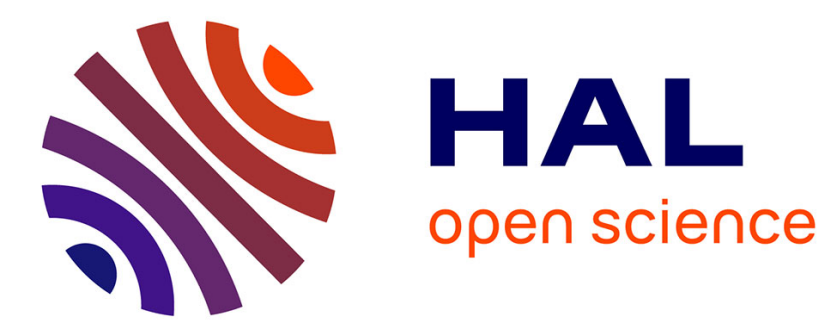

\title{
WULoRa: An Energy Efficient IoT End-Node for Energy Harvesting and Heterogeneous Communication
}

Michele Magno, Fayçal Ait Aoudia, Matthieu Gautier, Olivier Berder, Luca Benini

\section{- To cite this version:}

Michele Magno, Fayçal Ait Aoudia, Matthieu Gautier, Olivier Berder, Luca Benini. WULoRa: An Energy Efficient IoT End-Node for Energy Harvesting and Heterogeneous Communication. IEEE/ACM Design, Automation \& Test in Europe Conference \& Exhibition (DATE), Mar 2017, Lausanne, Switzerland. hal-01501163

\section{HAL Id: hal-01501163 \\ https://hal.science/hal-01501163}

Submitted on 3 Apr 2017

HAL is a multi-disciplinary open access archive for the deposit and dissemination of scientific research documents, whether they are published or not. The documents may come from teaching and research institutions in France or abroad, or from public or private research centers.
L'archive ouverte pluridisciplinaire HAL, est destinée au dépôt et à la diffusion de documents scientifiques de niveau recherche, publiés ou non, émanant des établissements d'enseignement et de recherche français ou étrangers, des laboratoires publics ou privés. 


\title{
WULoRa: An Energy Efficient IoT End-Node for Energy Harvesting and Heterogeneous Communication
}

\author{
Michele Magno ${ }^{1}$, Faycal Ait Aoudia ${ }^{2}$, Matthieu Gautier ${ }^{2}$, Olivier Berder ${ }^{2}$, and Luca Benini ${ }^{1}$ \\ ${ }^{1}$ ETH Zürich \\ ${ }^{2}$ IRISA, University of Rennes 1
}

\begin{abstract}
Intelligent connected objects, which build the IoT, are electronic devices usually supplied by batteries that significantly limit their life-time. These devices are expected to be deployed in very large numbers, and manual replacement of their batteries will severely restrict their large-scale or widearea deployments. Therefore energy efficiency is of the utmost importance in the design of these devices. The wireless communication between the distributed sensor devices and the host stations can consume significant energy, even more when data needs to reach several kilometers of distance. In this paper, we present an energy-efficient multi-sensing platform that exploits energy harvesting, long-range communication and ultra-low-power shortrange wake-up radio to achieve self sustainability in a kilometer range network. The proposed platform is designed with power efficiency in mind and exploits the always-on wake-up radio as both receiver and a power management unit to significantly reduce the quiescent current even continuously listening the wireless channel. Moreover the platform allows the building of an heterogeneous long-short range network architecture to reduce the latency and reduce the power consumption in listening phase at only 4.6 $\mu \mathrm{W}$. Experimental results and simulations demonstrate the benefits of the proposed platform and heterogeneous network.
\end{abstract}

\section{INTRODUCTION}

The Internet of Things (IoT) is an emerging technology domain that is rapidly growing. Today, sensing devices are entering applications such as assisted living, home automation, indoor and outdoor surveillance and monitoring, e-health, industrial manufacturing, etc. These intelligent devices are expected to work months and years but are usually supplied by batteries. The wireless communication between the distributed sensor devices and the host stations can be very costly in terms of energy consumption, especially in networks that have to span several kilometers. Therefore, the design of the wireless communication for a modern IoT node deserves special attention.

In recent years, advances in wireless technology enabled low-power Long Range (LR) communication. As an example, $\mathrm{LoRa}^{\mathrm{TM}}$ [1], by the LoRa ${ }^{\mathrm{TM}}$ Alliance, achieves power consumption similar to usual Wireless Sensor Networks (WSN) node transceivers [2] while transmitting to a distance of 10-20 kilometers, albeit at data rates typically smaller than $10 \mathrm{kbps}$. In this system, gateway nodes will always be listening to the channel and low latency can be achieved by using LoRa ${ }^{\mathrm{TM}}$ for uplink communications, from the nodes to the gateway. To save energy on the IoT nodes, a trade-off between latency (usually in the order of minutes or hours) and power consumption can be made for downlink communication, from the gateway to the nodes [1]. However, some applications such as industrial machine health monitoring or zone surveillance require both low-latency and low-power consumption at the same time [3].

Wake-up Receivers (WuRx) form another technology for energy efficiency that continuously monitors the channel while consuming orders of magnitude less power than traditional transceivers [4]. By enabling "pure" asynchronous communication [5], these devices wake up the entire system using interrupts only when a specific signal, called Wake-up Beacon $(\mathrm{WuB})$, is detected [6], [7]. Compared to traditional WSN transceivers [4], [8] WuRx are usually characterized by lower sensitivity and lower bit-rate, meaning they can only operate in Short Range (SR), i.e. tens of meters. Note that, WuRx and LoRa ${ }^{\mathrm{TM}}$ provide orthogonal features that are often required together in WSN applications.

Low-power and energy-efficient communication combined with other low-power design techniques [9], [10] significantly improve the life time of the battery-powered sensors. However, these alone are not sufficient to make IoT devices selfsufficient and such devices will need in-field battery replacement. Energy Harvesting (EH) technology, which is able to collect and store energy from environmental sources (i.e., light, vibration, difference of temperature) can in many cases be used to overcome this limitation.

In this paper we present a sensor node that combines long and short range commutation exploiting the emerging LoRa ${ }^{\mathrm{TM}}$ and wake-up radio designed to work only with energy harvester. We adopt a combination of hardware and software techniques to improve the overall energy efficiency of the proposed solution. The sensor platform hosts five different sensors and it features highly-efficient power-management and conversion design for solar and thermal energy harvesting. The major contributions of the paper are:

- An energy-efficient platform that enhances the functional lifetime of indoor and outdoor sensing applications exploiting ultra-low power hardware architecture, $\mathrm{EH}$, heterogeneous long-short range communication and WuRx technology. A multi-source $\mathrm{EH}$ sub-system is designed and the wake-up radio is used as a "wireless trigger" for enhancing the power management capabilities of the node.

- An energy-efficient network architecture supporting long-range communication and ultra-low-power wakeup radio receiver to reduce both power consumption and latency. 
- Quantitative, empirical validation of the proposed approach via simulation and experimentation to highlight the benefits of the proposed combination.

The rest of the paper is organized as follows. Existing works on hardware platforms using several radios are summarized in Section II. The heterogeneous network architecture described in Section III legitimates our platform design detailed in Section IV. This platform is experimentally evaluated in Section V and analytic results are given in Section VI, before a conclusion is drawn in Section VII.

\section{RELATED WORK}

Given the variety of applications they have to handle, most of modern wireless devices used for wireless sensor networks embed several radio modules to reduce energy consumption and latency for neighbor discovery and opportunistic networking [11]. While using multiple radio systems increases both the size and price of devices, relying on the lowpower radio to reduce the utilization of the high-power radio subsystem can ultimately reduce power consumption of the whole system. Most of related works propose to combine Wi-Fi (IEEE 802.11) and Zigbee (IEEE 802.15.4) for power saving [12], [13]. In these works, the low-level radio is used to discover, configure and activate a high-level radio link when a connection is needed. The authors in Pering et al. [14] experimented on a platform that provides Bluetooth, Zigbee and $\mathrm{Wi}-\mathrm{Fi}$, and revealed that the lower power consumption is achieved by employing Zigbee and Wi-Fi. However, using Bluetooth and Zigbee as a low power radio is still too costly for WSN applications, which is the focus of this work. Moreover, none of these standards is able to achieve kilometer-range communication.

Several radio technologies (e.g Sigfox, LoRa ${ }^{\mathrm{TM}}$, Weightless, NB-IoT ...) are currently promising to fill the gap in low-power Device-to-Device (D2D) communications over long distances [15]. In LoRa ${ }^{\mathrm{TM}}$ network architecture [1], all WSN nodes communicate directly with the gateway, which serves as a bridge between the nodes and a network server. This gateway is always actively listening to the channel. Three types of classes are defined for end-devices: A, B and C. Class A is the lowest power consuming class where nodes only leave the sleep state to send their data. Each uplink transmission is followed by two short downlink receive windows. Therefore, transmission slot is scheduled by the device based on its own communication needs. The downlink communication will require to wait the next scheduled uplink transmission. Class B devices open additional receive windows at scheduled times in addition to class A receive windows, and time synchronized beacons from the gateway are used to allow the gateway to know when devices are listening. Finally, class $\mathrm{C}$ devices are continuously listening, except when they are transmitting. Therefore, using the LoRa ${ }^{\mathrm{TM}}$ network architecture, a tradeoff can be made between latency and energy consumption for downlink communication. In this work, we try to reduce this gap using a orthogonal highly efficient technology for shortrange communication (WuRx).

The authors in [16] propose the OpenMote+ platform, targeting industrial applications, combining three communication modules: one for LR (kilometers-range) communication,

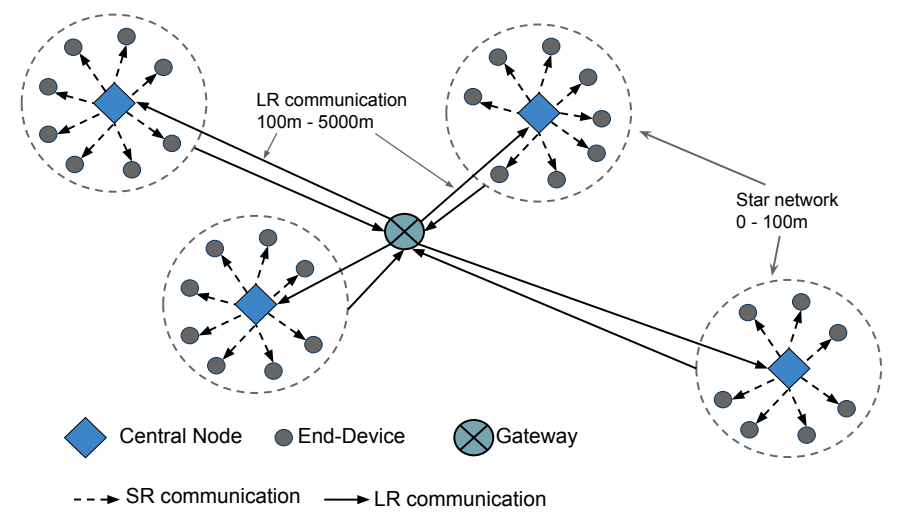

Fig. 1: Heterogeneous network architecture.

one for hundreds of meters communication, and one for contact-based communication. In this work, different radios are used as independent wireless communication, while we propose a collaborative combination of two technologies. In addition, we also propose to use WuRx to eliminate idle listening and perform purely asynchronous communication for SR communications. In [9] a platform that incorporates both energy harvesting and wake-up radio has been presented. In the proposed work, we extend this concept by adding longrange communication and using the wake-up radio also for low-power energy management. Finally, in [17] the authors have presented a preliminary study of the benefits of using a system with short-long range radio using a collaborative protocol with LoRa ${ }^{\mathrm{TM}}$ and WuRx, showing that using WuRx can both decrease latency and increase energy efficiency. In our work, the long-short range concept is significantly extended using the WuRx as power manager to further increase the energy efficiency and platform has been designed including also EH and sensors to evaluate the benefits of the proposed approach with experimental results.

\section{NETWORK ARCHITECTURE}

Many applications, such as connected farms, zone surveillance or even smart factories require both local short-range communications (within a building, or on a field) and longrange communications between each of these areas, leading to a heterogeneous and clusterized network, as illustrated by Fig. 1. The sensor nodes are organized in star topology clusters, where several End Devices (EDs) are connected with a Central Node $(\mathrm{CN})$ in a range of tens of meters. These $\mathrm{CN}$ are in charge to communicate with both the EDs and a remote host (gateway) that can be at a distance of several kilometers.

To achieve such a network architecture with a single longrange radio such as $\mathrm{LoRa}^{\mathrm{TM}}$ a trade-off has to be made between latency and energy consumption. Using LoRa ${ }^{\mathrm{TM}}$ in class $\mathrm{C}$ would allow EDs to stay awake permanently, allowing reasonable latency for downlink communications from the $\mathrm{CN}$ but at the price of important waste of energy. On the other hand, class A operation is far less energy hungry but latency becomes detrimental. Therefore combining long-range and wake-up radio solutions offers a good compromise.

In the proposed network, each ED embeds both a LoRa module and a low-power always-on WuRx. The main advantage of such an architecture is that the $\mathrm{CN}$ or another 


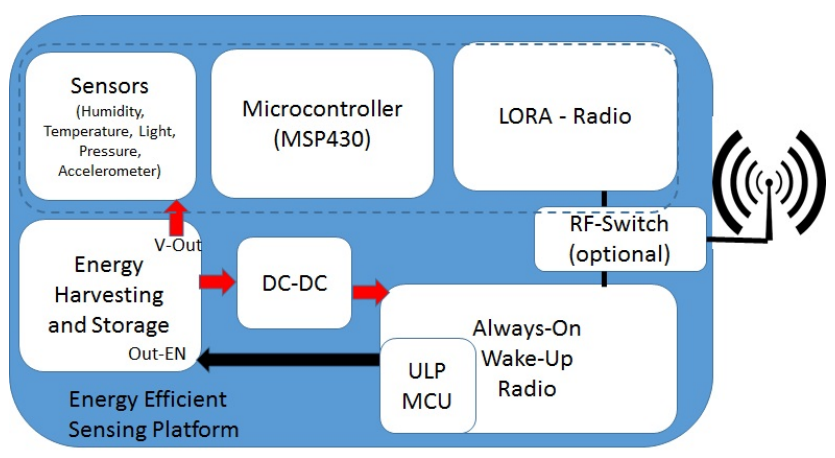

Fig. 2: Block diagram of the designed sensor node.

ED can wake up one or more EDs by sending WuBs even when the LoRaTM radio in the receiver node is off to save energy. There are three cases when an ED is awake: when an interrupt is triggered on a timer, an event from the environment or the reception of a WuB. The first two events may lead to a data message sent through the gateway. In that case, the EDs send the data directly to the gateway using LR communication, as shown by Fig. 1. The other possibility is when the gateway sends a command to one or more EDs. The gateway transmits the command to the $\mathrm{CN}$, which then forwards the command to the addressed EDs, by first waking up the addressed EDs by sending a WuB which is received by the WuRx of teh ED. The $\mathrm{CN}$ and the gateway communicate using the standard LoRa $^{\mathrm{TM}}$ scheme [1]. Hence, direct LR communication is unidirectional between the EDs and the gateway, while it remains bidirectional between the gateway and the $\mathrm{CN}$.

\section{PlatForm Design}

The block diagram of the designed sensor node is shown in Fig. 2. The main aim of the system is to have a multi-sensor platform that can be used for different indoor and outdoor applications. In Fig. 2, main blocks can be identified: an ultra low-power micro-controller MSP430 from Texas Instruments; sensors; long-range radio based on LoRa ${ }^{\mathrm{TM}}$; energy harvesting circuit which provides the regulated voltage for all the subsystems except the always-on wake-up radio. The wake-up radio is supplied by a dedicated DC-DC converter. In this way, it is possible to use the ultra low-power micro-controller embedded in the WuRx also as a power manager for the whole system to control the power state of the entire platform. In the following subsection we present more in details the blocks.

\section{A. Sensors Subsystem}

The sensing block consists of five Micro-electromechanical-system (MEMS) sensors, a light sensor, a humidity sensor a temperature sensor, a barometer and finally an Inertial Measurement Unit (IMU). All the sensors are connected to the micro-controller trough an I2C serial interface. Table I summarizes the features of the sensors and the targeted applications.

\section{B. Energy harvesting, storage monitor and power subsystem}

Converting energy from the generator to the storage is crucial for any energy harvesting circuit. In recent years, many manufacturers have provided integrated circuits (IC) specific

\begin{tabular}{c||c|c|c|c}
\multirow{2}{*}{ Sensors } & References & \multicolumn{2}{|c|}{ Current consumption } & \multirow{2}{*}{ Applications } \\
\cline { 3 - 4 } & Acquisition & Sleep & \\
\hline \hline Light & ISL29023 & $70 \mu \mathrm{A}$ & $300 \mathrm{nA}$ & $\begin{array}{c}\text { Home automation } \\
\text { Weather monitoring }\end{array}$ \\
\hline $\begin{array}{c}\text { Humidity } \\
\& \text { temperature }\end{array}$ & SHT21 & $270 \mu \mathrm{A}$ & $135 \mathrm{nA}$ & $\begin{array}{c}\text { Agriculture } \\
\text { Weather monitoring }\end{array}$ \\
\hline $\begin{array}{c}\text { Barometric } \\
\text { pressure }\end{array}$ & BMP180 & $27 \mu \mathrm{A}$ & $\mathrm{n} / \mathrm{a}$ & $\begin{array}{c}\text { Localization } \\
\text { Weather monitoring }\end{array}$ \\
\hline $\begin{array}{c}\text { Infrared } \\
\text { temperature }\end{array}$ & TMP006 & $270 \mu \mathrm{A}$ & few nA & $\begin{array}{c}\text { Zone surveillance } \\
\text { Structure } \\
\text { monitoring }\end{array}$ \\
\hline IMU & MPU-915 & $<4 \mathrm{~mA}$ & $1 \mu \mathrm{A}$ & $\begin{array}{c}\text { WSBAN } \\
\text { Localization }\end{array}$
\end{tabular}

TABLE I: Sensor features.

for energy harvesting. Our circuit is designed around the stateof-the-art BQ25570 from Texas Instruments, which allows a conversion efficiency of $90 \%$ and provides maximum power point tracking to maximize the efficiency in all the operative conditions. The BQ25570 has very low start-up voltage of only $330 \mathrm{mV}$. This value is sufficient for indoor solar panels and thermometric transducers that usually have low output voltage. Finally the IC can recharge both supercapacitors and Li-Ion Battery with the same efficiency. In addition, the IC provides a tuneable buck converter to supply the rest of the circuit directly. We used a 3V voltage for our prototype. The BQ25570 offers the possibility to enable and disable the buck converter via an enable pin. To maximize the energy efficiency we control this pin trough the the always-on ultra low-power wake-up radio that is supplied from another buck converter, reducing the quiescent current in sleep mode significantly.

Knowing how much energy is available in the storage is vital for the power management to optimize the use of the residual energy optimally. With this in mind we inserted an accurate $(0.25 \%)$ and low power (only $4 \mu \mathrm{W}$ in low power mode) fuel gauge. The STC3115 from STMicroelectronics has been connected trough the I2C serial port to the MSP430 so that it can determine the exact level of the battery at any time.

A dedicated low power DC-DC buck converter TPS62736 from Texas Instruments optimized for low current output is used to power the always on region of the system that includes the wake-up radio. This converter achieves up to $95 \%$ efficiency and it has a very low quiescent current in active mode of only $300 \mathrm{nA}$.

\section{Radio Subsystem}

1) SX1276 module: long-short range communication: The SX1276 transceiver from Semtech incorporates (G)FSK and OOK modulations (needed for the wake up radio), as well as the LoRa ${ }^{\mathrm{TM}}$ physical layer. Thus, the designed platform has long range and short range communication using a single transceiver configured during run-time by the micro-controller. The SX1276 module can be switched between different modulation schemes, allowing different modulation approaches to co-exist. With $\mathrm{LoRa}^{\mathrm{TM}}$, it is theoretically possible to reach a range of $22 \mathrm{~km}$, with a data rate of around $10 \mathrm{kbps}$ in the $868 / 915 \mathrm{MHz}$ ISM bands [1], [15]. Using the long-range communication allows sending data to far away sink nodes that can collect the data from the sensors distributed in the field [17]. The OOK modulation of the SX1276 is used to send $\mathrm{WuB}$ and commands to the WuRx. This module is the 
only module needed as transmitter for both short- and longrange communication. Moreover when two ED within short distance wants to communicate with each other, the SX1276 is configured to use GFSK modulation increasing the energy efficiency of the communication during the data transmission.

2) Wake-up Radio: In addition to the SX1276 transceiver, each sensor node is equipped with an instance of the WuRx designed in [8], which receives OOK modulated data. The WuRx has a sensitivity of $-55 \mathrm{dBm}$ and incorporates an ultra low-power micro-controller (ULP MCU) that provides computational resources to the WuRx and interface with the main micro-controller. The wake-up radio has been tuned to $868 \mathrm{MHz}$ ISM band to receive data from the SX1276 module [8]. The ULP MCU is a PIC12LF1552 from Microchip configured to be awakened when a WuB is detected from the $\mathrm{RF}$ front-end. Address matching is provided allowing only specific nodes to wake up. Moreover, the ULP MCU in the wake-up radio subsystem has been used as a energy manager in charge to switch on and off the main Buck-converter embedded in the BQ25570. This allows the whole platform to go in a deep sleep mode and be woken up from wireless beacon or at fixed time intervals (duty cycling) by the ULP MCU according to power policies implemented at application level.

\section{Main micro-controller}

The energy efficient platform employs the ultra low-power TI MSP430FR5969 micro-controller (16-bit processor, with 64 KB FRAM non-volatile RAM, 12-bit ADC, op-amps and serial interfaces interfaces). The micro-controller is connected with the sensors and the radio module and it executes programmable power management policies, data collection from sensors and sending data or commands with the SX1276 module (using LoRa ${ }^{\mathrm{TM}}$ or $\mathrm{OOK}$ modulation). The microcontroller has adequate computational resources to guarantee the best energy efficiency. The selection of the micro-controller has been primarily driven by the ultra low-power consumption $(<1 \mu \mathrm{W}$ in sleep mode) and due to the presence of the embedded on-board non-volatile RAM. The non-volatile memory is particularly useful when power is completely switched off as all the status registers are saved in the FRAM to switch-off and switch-on the platform without lose the status. This is a well-know technique [18] to save energy and in our platform is controlled by the ULP MCU within the WuRx.

\section{EXPERIMENTAL CHARACTERIZATION}

For the power consumption measurements of the platform (Fig. 3), the Keysight N6705B DC Power Analyzer was used. The current and voltage have been measured on the battery input setting the power analyzer at the fixed voltage of $3.7 \mathrm{~V}$, the nominal voltage of common rechargeable Li-Ion batteries. With this setup the measurements take into account all the losses due to the power conversion, quiescent current, and the effective power consumed from the battery is evaluated. For the evaluation, the MSP430 has been set to $1 \mathrm{MHz}$ and the PIC micro-controller to $2 \mathrm{MHz}$ when in active modes.

Table II shows the power consumption of the most significant operation modes. The first two rows demonstrate the low quiescent current achievable. In all modes, the wake-up radio is always on and the energy harvesting can charge the battery

\begin{tabular}{|c|c|}
\hline Modes & Power \\
\hline Energy Harvesting + WuRx (Listening) & $4.6 \mu \mathrm{W}$ \\
\hline Energy Harvesting + WuRx (Receiving - PIC ON) & $314.5 \mu \mathrm{W}$ \\
\hline $\mathrm{EH}+\mathrm{WuRx}+\mathrm{MPS} 430(\mathrm{LPM} 3)+\mathrm{LoRa}^{\mathrm{TM}}$ Sleep & $0.65 \mathrm{~mW}$ \\
\hline $\mathrm{EH}+\mathrm{WuRx}+\mathrm{MSP} 430(\mathrm{LPM} 3)+\mathrm{LoRa}^{\mathrm{TM}} \mathrm{RX}$ & $51.8 \mathrm{~mW}$ \\
\hline MSP430 (active) + Acquistion from SHT21 + LoRaTM Sleep & $8.14 \mathrm{~mW}$ \\
\hline $\mathrm{MSP}$ (Active) $+\mathrm{TX} \mathrm{LoRa}{ }^{\mathrm{TM}}+14 \mathrm{dBm}$ & $381.1 \mathrm{~mW}$ \\
\hline MSP (Active) $+\mathrm{TX} \mathrm{LoRa}{ }^{\mathrm{TM}}+10 \mathrm{dBm}$ & $281.2 \mathrm{~mW}$ \\
\hline MSP (Active) $+\mathrm{TX} \mathrm{LoRa}{ }^{\mathrm{TM}}+5 \mathrm{dBm}$ & $203.5 \mathrm{~mW}$ \\
\hline MSP (Active) + TX LoRa ${ }^{\mathrm{TM}}+0 \mathrm{dBm}$ & $170.2 \mathrm{~mW}$ \\
\hline
\end{tabular}

TABLE II: Power measurement of the platform in different modes.

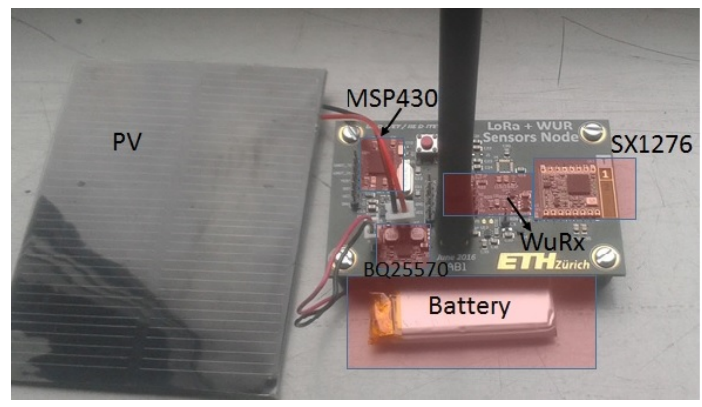

Fig. 3: Prototype of the sensing platform.

provided the environmental conditions (solar or thermal) allow it. When data is received from the WuRx the PIC microcontroller switches to active mode and the power consumption increases (row 2). With only less than $5 \mu \mathrm{W}$ (row 1) the sensor node can still receive wireless commands and perform power management using the PIC. The table also shows the power saving in sleep mode achieved by the use of the WuRx as power manager. In fact looking at row 2 , it can be seen that when the buck converter is active, also in sleep mode, the power of the platform rises up to $0.65 \mathrm{~mW}$ mainly due to the sum of the quiescent currents of all the components $\left(\mathrm{LoRa}^{\mathrm{TM}}\right.$, Sensors, battery monitor, buck converter). Another important information of the power measurements comes from the power consumption of $\mathrm{LoRa}^{\mathrm{TM}}$ in transmission (up $380 \mathrm{~mW}$ ) and especially in receiving mode (more than $50 \mathrm{~mW}$ ). In fact, the table highlights that using the $\mathrm{LoRa}^{\mathrm{TM}}$ module to receive continuously is unfeasible since its power is an order of magnitude higher than the WuRx. This demonstrate the benefit of the WuRx that consumes only $4.6 \mu \mathrm{W}$ to continuously listen to the channel.

To evaluate the range achievable for the short range communication, we perform several tests both in indoor and outdoor scenarios (line-of-sight), TABLE III shows the achievable range using $+10 \mathrm{dBm}$ and $+14 \mathrm{dBm}$ power output for the transmitter. The range has been evaluated using a $+3 \mathrm{dBi}$ antenna in both receiver and transmitter node. The same module SX1276 has been configured to transmit 16 bits at $1 \mathrm{kbps}$ in OOK communication. The tables shows that it is possible to achieve up to $55 \mathrm{~m}$ in indoor and $40 \mathrm{~m}$ in outdoor. These values are suitable for a wide range of applications as these are the common value of WSN applications. Finally, the time to send 


\begin{tabular}{c|c|c} 
OOK+WuRx & Power & Range \\
\hline \hline MSP430 (Active) SX1276 OOK $+14 \mathrm{dBm}$ & $120.5 \mathrm{~mW}$ & $55 \mathrm{~m}$ (Indoor) \\
\hline MSP430 (Active) SX1276 OOK $+14 \mathrm{dBm}$ & $120.5 \mathrm{~mW}$ & $40 \mathrm{~m}$ (Outdoor) \\
\hline MSP430 (Active) SX1276 OOK $+10 \mathrm{dBm}$ & $87 \mathrm{~mW}$ & $35 \mathrm{~m}$ (Indoor) \\
\hline MSP430 (Active) SX1276 OOK $+10 \mathrm{dBm}$ & $87 \mathrm{~mW}$ & $40 \mathrm{~m}$ (Outdoor)
\end{tabular}

TABLE III: Power consumption vs range when the SX1276 module is configured in OOK modulation.

16 bit packet has been measured for OOK and $\mathrm{LoRa}^{\mathrm{TM}}$ protocol and this results in $600 \mathrm{~ms}$ for $\mathrm{LoRa}^{\mathrm{TM}}$ and only $160 \mathrm{~ms}$ for OOK. This is due to the overhead that LoRa ${ }^{\mathrm{TM}}$ adds from the protocol, that makes WuRx using OOK communication more suitable for short-range and short message communication.

\section{ANALYTICAL EVALUATION}

The average rate at which commands are received by the EDs is denoted $\lambda_{r}$ while $\lambda_{g}$ is their packet transmission rate. $e_{r}$ and $e_{g}$ represent the energy cost to receive a command and send a packet respectively. Using the proposed platform, $e_{r}$ depends on the type of radio while $e_{g}$ is the same for all radios. The average power consumption $P_{C}$ is thus:

$$
P_{C}=\lambda_{r} e_{r}+\lambda_{g} e_{g}+P_{0},
$$

with $P_{0}$ a power overhead that depends on the radios and their associated MAC protocol.

For LoRa ${ }^{\mathrm{TM}}$ and IEEE $802.15 .4 /$ GFSK modulations, the overhead is due to periodic wake-up to listen to the channel and is equal to:

$$
P_{0}=\beta P_{\mathrm{Rx}},
$$

with $\beta$ the duty cycle ratio. For WuRx-based communication (OOK modulation), the overhead is due to the always listening mode and to false wake-ups of the PIC that occur in noisy environments. The power overhead is computed by:

$$
P_{0}=P_{\text {WuRx_listen }}+\alpha P_{\text {WuRx_PIC }} \text {, }
$$

with $\alpha$ the time ratio the PIC is ON while not receiving any WuB.

In the simulation, $\lambda_{r}$ and $\lambda_{g}$ are set to $\frac{1}{60} \mathrm{~Hz}$ and $\frac{1}{10} \mathrm{~Hz}$. In order to explore different use cases, two payload lengths are defined: SHORT PL for 10 Bytes and LONG PL for 100 Bytes, two duty cycles: Low DC for $\beta=0.1 \%$ and HIGH DC for $\beta=0.5 \%$ and two false wake-up factors: Low FW for $\alpha=0 \%$ and HIGH FW for $\alpha=20 \%$.

The range of each radio access is computed using the Logdistance path loss model. The channel attenuation $P L$ is given as a function of the range $d$ and the path loss exponent $\gamma$ that characterized the propagation conditions.

$$
P L=P L_{0}+10 \gamma \log \left(\frac{d}{d_{0}}\right) .
$$

$P L_{0}$ is the path loss at the reference distance $d_{0}$ and is equal to $31 \mathrm{~dB}\left(d_{0}=1 \mathrm{~m}\right)$ in the $868 \mathrm{MHz}$ frequency band. The range is finally estimated so that $P_{T}-P L$ is higher than the radio sensitivity $S_{\text {ref }}$.

$S_{\text {ref }}$ was measured to $-55 \mathrm{dBm},-105 \mathrm{dBm}$ and $-128 \mathrm{dBm}$ for the WuRx, the GFSK and the LoRa ${ }^{\mathrm{TM}}$ radio respectively. $\gamma$ is

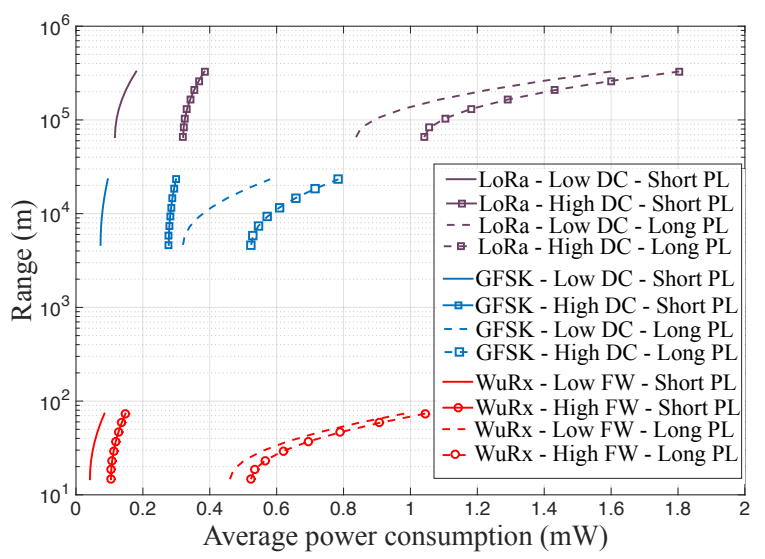

Fig. 4: Theoretical range of different radio access as a function of the power consumption.

set to 2.8 and the transmission power $P_{T}$ are set between 0 to $14 \mathrm{dBm}$ as in TABLE II.

For duty-cycle-based communication, the gateway has to wait that the ED opens a receiving slot to send commands. Thus the average latency of command transmission is:

$$
L_{D C}=\frac{D}{2 \beta},
$$

with $D$ the duration of the receiving slot that depends on the radio (i.e. LoRa ${ }^{\mathrm{TM}}$ or GFSK).

Using WuRx, the transmission is purely asynchronous. Thus, the latency $L_{W u R x}$ of a downlink transmission is equal to the time required for a packet transmission.

In the simulation, $D$ is equal to $1.2 \mathrm{~ms}$ at $6.84 \mathrm{kbit} / \mathrm{s}$ for Lora $^{\mathrm{TM}}$ and $0.4 \mathrm{~ms}$ at 20kbit/s for GFSK [17]. $L_{W u R x}$ depends on the payload length previously mentioned.

The communication schemes allowed by the platform were analytically compared using the previous models and the measurements performed in Section V. Fig. 4 shows the theoretical range of the different radios and configurations as a function of the power consumption of the ED. The latencies of the different radio technologies are compared in Fig. 5 as a function of the duty cycle ratio.

The first remark is that the range of each radio is clearly different. We can also notice the huge difference in power consumption between $\mathrm{LoRa}^{\mathrm{TM}}$ and WuRx. The main dilemma is choosing between GFSK and WuRx for short-range communications. Indeed, GFSK power consumption outperforms

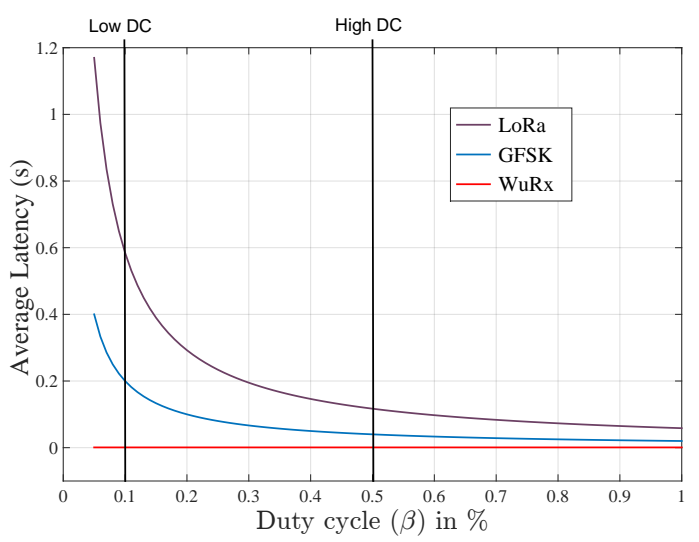

Fig. 5: Average latency of the different radio access as a function a the duty cycle $\beta$. LONG PL is considered for the WuRx. 


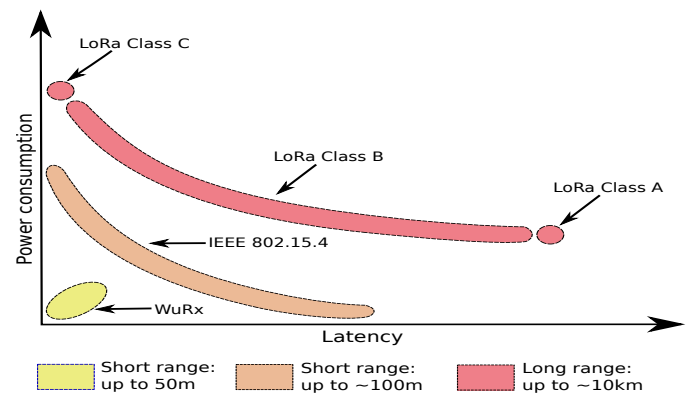

Fig. 6: Latency-Power-Range trade-off achievable by the platform.

WuRx for LONG PL while WuRx is more efficient for SHORT PL case (which is the most current in IoT applications). The choice of the duty cycle is also a key issue for GFSK and LoRa ${ }^{\mathrm{TM}}$ radios. Fig. 4 shows that configuration LOW DC can significantly reduce the power consumption. However, as shown on Fig. 5, Low DC leads to increased latency. WuRx communication achieves both very low latency and low power consumption, making this solution the winning one for SHORT PL applications. Finally, we can notice the low influence of the false alarm factor $\alpha$ on the power consumption of the WuRx. Even for $20 \%$ of false alarm, the ultra-low power PIC wakeups have a small impact on the average power consumption.

Fig. 6 summarizes the use of the different radios according to requirements of the applications. LoRa ${ }^{\mathrm{TM}}$ class $\mathrm{A}$ is equivalent to low DC as the node only wakes up to send data. On the contrary, the node is always awake in class $\mathrm{B}(\beta=1)$. Class B and GFSK modulation use DC protocols with periodic sleep and awake of the node. A Pareto front is achieved with these technologies with a trade-off between latency and power consumption. For short range communications, asynchronous communication using WuRx outperforms the other communication in both latency and power consumption. Due to the presence of both short-long range communication and the ultra low power listening mode, the proposed solution can work with very low power waiting for beacons and achieve $\mu \mathrm{W}$ deep sleep mode. The overall energy efficiency is achieved reducing the activity of the long-range one only when necessary, and using the short range and wake up radio for the other communications.

\section{CONCLUSION}

The recent development of utilizing complementary radio technologies, namely long-range and wake-up radios, opens new perspectives for D2D network architectures, since achieving information through energy autonomous nodes with reasonable latency is now possible, even for downlink transmission. In this paper, we propose an sensor platform architecture combining $\mathrm{LoRa}^{\mathrm{TM}}$, wake-up radio designed for self-sustaining sensing application. To achieve this aim several low-power design and techniques such as using Wake up radio as an energy management have been used especially to increase the communication efficiency and reduce the power in sleep mode. By collaboratively combining two radios, an heterogeneous network is presented. We demonstrate the benefits of our approach with experimental platform characterizations, conducted to extract features in terms of power consumption and range. These measurements were used then to feed an analytical model.

\section{ACKNOWLEDGMENT}

This work was supported by "Transient Computing Systems" (200021_157048), and by "POMADE" project, funded by $\mathrm{CD} 22$ and the Brittany region.

\section{REFERENCES}

[1] "LoRaWANTM, Specification v1.0, LoRa Alliance, Inc. 2400 Camino Ramon, Suite 375 San Ramon, CA 94583 (2015),' LoRa Alliance, Tech. Rep., 2015.

[2] B. Martinez, M. Monton, I. Vilajosana, and J. Prades, "The Power of Models: Modeling Power Consumption for IoT Devices," IEEE Sensors Journal, vol. 15, no. 10, pp. 5777-5789, October 2015.

[3] T. Zheng, M. Gidlund, and J. kerberg, "WirArb: A New MAC Protocol for Time Critical Industrial Wireless Sensor Network Applications," IEEE Sensors Journal, vol. 16, no. 7, pp. 2127-2139, April 2016.

[4] N. S. Mazloum and O. Edfors, "Performance Analysis and Energy Optimization of Wake-Up Receiver Schemes for Wireless Low-Power Applications," IEEE Transactions on Wireless Communications, vol. 13, no. 12, pp. 7050-7061, December 2014.

[5] F. Ait Aoudia, M. Gautier, and O. Berder, "OPWUM: Opportunistic MAC Protocol Leveraging Wake-Up Receivers in WSNs," Journal of Sensors, January 2016, Article ID 6263719.

[6] D. Spenza, M. Magno, S. Basagni, L. Benini, M. Paoli, and C. Petrioli, "Beyond Duty Cycling: Wake-up Radio with Selective Awakenings for Long-Lived Wireless Sensing Systems," in IEEE INFOCOM, April 2015, pp. 522-530.

[7] H. Yomo, K. Abe, Y. Ezure, T. Ito, A. Hasegawa, and T. Ikenaga, "Radio-On-Demand Sensor and Actuator Networks (ROD-SAN): System Design and Field Trial," in IEEE Global Communications Conference (GLOBECOM), December 2015, pp. 1-6.

[8] M. Magno, V. Jelicic, B. Srbinovski, V. Bilas, E. Popovici, and L. Benini, "Design, Implementation, and Performance Evaluation of a Flexible Low-Latency Nanowatt Wake-Up Radio Receiver," IEEE Transactions on Industrial Informatics, vol. 12, no. 2, pp. 633-644, April 2016.

[9] M. Magno, S. Marinkovic, D. Brunelli, E. Popovici, B. O’Flynn, and L. Benini, "Smart power unit with ultra low power radio trigger capabilities for wireless sensor networks," in Proceedings of the Conference on Design, Automation and Test in Europe, ser. DATE '12. San Jose, CA, USA: EDA Consortium, 2012, pp. 75-80.

[10] A. A. Aziz, Y. A. Sekercioglu, P. Fitzpatrick, and M. Ivanovich, "A survey on distributed topology control techniques for extending the lifetime of battery powered wireless sensor networks," IEEE Communications Surveys Tutorials, vol. 15, no. 1, pp. 121-144, First 2013.

[11] R. Pozza, M. Nati, S. Georgoulas, K. Moessner, and A. Gluhak, "Neighbor Discovery for Opportunistic Networking in Internet of Things Scenarios: A Survey," IEEE Access, vol. 3, pp. 1101-1131, 2015.

[12] H. Qin and W. Zhang, "Zigbee-assisted power saving management for mobile devices," IEEE Transactions on Mobile Computing, vol. 13, no. 12, pp. 2933-2947, Dec 2014.

[13] R. Zhou, Y. Xiong, G. Xing, L. Sun, and J. Ma, "ZiFi: Wireless LAN Discovery via ZigBee Interference Signatures," in Annual International Conference on Mobile Computing and Networking. ACM, 2010.

[14] T. Pering, V. Raghunathan, and R. Want, "Exploiting radio hierarchies for power-efficient wireless device discovery and connection setup," in 18th Int. Conference on VLSI Design, January 2005, pp. 774-779.

[15] C. Goursaud and J.-M. Gorce, "Dedicated networks for IoT : PHY / MAC state of the art and challenges," EAI endorsed transactions on Internet of Things, October 2015.

[16] P. Tuset-Peiró, X. Vilajosana, and T. Watteyne, "OpenMote+: a RangeAgile Multi-Radio Mote," in Proceedings of the International Conference on Embedded Wireless Systems and Networks, 2016, pp. 333-334.

[17] F. A. Aoudia, M. Magno, M. Gautier, O. Berder, and L. Benini, "A low latency and energy efficient communication architecture for heterogeneous long-short range communication,", in Euromicro Conference on Digital System Design (DSD), August 2016, pp. 1-6.

[18] A. Gomez, L. Sigrist, M. Magno, L. Benini, and L. Thiele, "Dynamic energy burst scaling for transiently powered systems," in Design, Automation Test in Europe Conference Exhibition (DATE), March 2016, pp. 349-354. 